\title{
MICROMECHANICAL TIME DELAY MECHANISMS FOR ORDNANCE FUSING
}

\author{
Jing Liu and Don L. DeVoe \\ Center for Micro Engineering, University of Maryland \\ College Park, MD USA
}

\begin{abstract}
Micromechanical SOI/DRIE time delay mechanisms have been developed as part of a larger fuzing system for rifled munitions, in which a passive timing mechanism triggers at a predetermined rotational speed, followed by a desired delay time before the next element of the munition fuzing train is activated. Mechanism arrays designed to initiate at centrifugal accelerations up to $263 \mathrm{~g}$ are simulated and characterized using a high-speed camera, with delay times of between 0.67 and $0.95 \mathrm{~ms}$ achieved for single array elements.
\end{abstract}

\section{INTRODUCTION}

Mechanical time delay mechanisms for munition applications typically initiate after a specific launch condition is met (e.g. threshold barrel velocity). Arming of the munition occuring once the set delay time has passed, ensuring that the munition is sufficiently far from the barrel to prevent premature detonation during the launch cycle [1]. Using a purely mechanical delay mechanism provides several advantages over electronic timers, including robustness to electronic interference and jamming, and passive operation without the need for external power. Microfabrication technology offers important benefits for time delay mechanisms, including small size, light weight, and low perunit cost. Microfabricated inertial switches have been used in fuzing applications [2,3], but these devices are designed to trigger instantaneously upon reaching a threshold acceleration rather than providing a post-threshold delay.

A range of timing mechanisms which release stored spring energy have been reported, including runaway escapements [4-6]], gearless runaway escapements $[7,8]$, and tuned escapements [911]. A microfabricated escapement mechanism for fuzing applications was recently described using both SOI/DRIE and LIGA methods [12] based on a pre-tensioned spring to store energy prior to releasing a slotted peg. In contrast to these designs which use stored potential energy to drive the mechanisms, a different design based on a rotary sequential-leaf mechanism $[1,13]$ is used here. This design consists of an array of identical elements that pivot around their individual rotation centers. Each element is constrained from rotating until the previous element in the array has rotated past a critical angle. The first element in the chain is designed to rotate through the critical angle only after a linear acceleration threshold has been reached. When the first device element reaches its critical angle, the second element is allowed to move. The dynamics of the second element defines a time period during which the element travels from its initial position through its own critical angle, and so on until all elements of the array have switched. The total delay time provided by the mechanism is the summation of individual delay times for each element in the chain.

The sequential-leaf design is particularly attractive for miniaturization. Unlike other types of time delay mechanisms, the

\author{
Lawrence Fan \\ Naval Surface Warfare Center \\ Indian Head, MD
}

leaf mechanism can be realized using an array of identical structures, each consisting of a simple mass and rotational suspension spring. Fabrication is compatible with single-layer SOI/DRIE processing. Since the design does not rely on stored energy, no post-fabrication assembly is required.

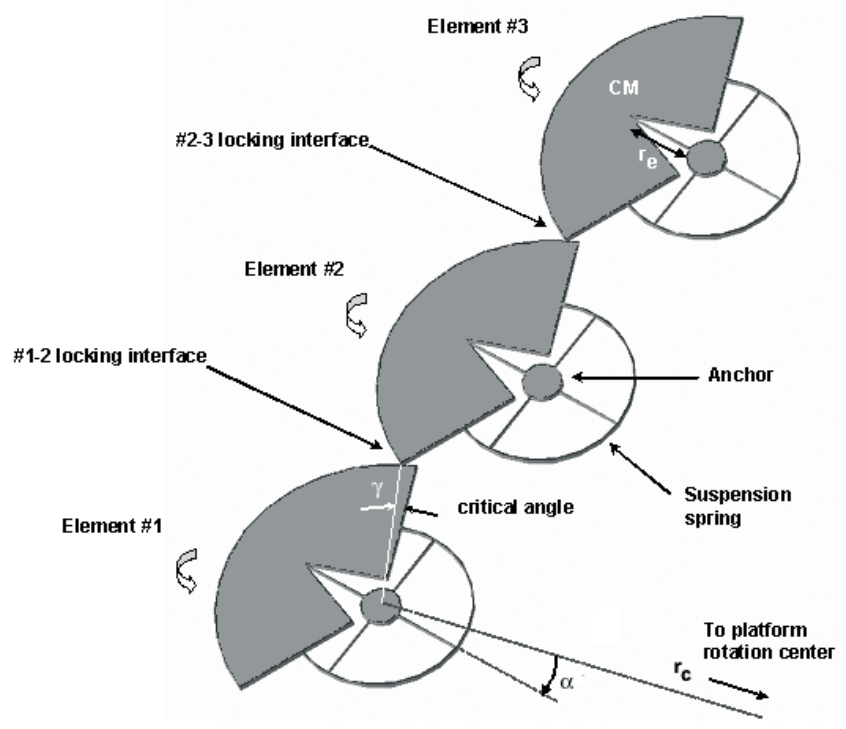

Fig. 1 Schematic of a 3-element rotary sequential-leaf time delay mechanism.

While macro-scale leaf mechanisms have been designed to respond to linear accelerations [14,15], the concept is extended in this paper to work with centrifugal acceleration. A schematic layout of a 3-element design is shown in Fig. 1. Each element consists of a fan-shaped seismic mass suspended above the substrate by a rotational spring composed of a rigid ring structure and four beams which share a common anchor point. The center of mass is designed to coincide with the apex of the v-notch where one of the quad-symmetric anchor beams connects to the mass.

\section{THEORY}

Consider a single leaf element as part of the 3-element mechanism shown in Fig. 1. The mechanism is secured to a platform rotating at a constant angular velocity of $\Omega$, with each leaf possessing an anchor point located a distance $r_{c}$ from the rotational center of the platform. When the platform rotates, centrifugal force creates a moment which induces a rotation of the first leaf about its anchor. The nonlinear equation of motion for a single leaf element is readily derived using a Lagrangian approach as,

$\ddot{\theta}\left(m r_{e}^{2}+I\right)-\Omega^{2} m r_{c} r_{e} \sin (\theta+\alpha)+k(\theta) \theta+T(\theta, \dot{\theta})=0$

where $\theta$ is the element rotation from its initial equilibrium position, $\mathrm{m}$ is the mass of the mechanism element, $\mathrm{I}$ is the element's moment of inertia about its center of mass, and $\mathrm{k}$ is the 
torsional spring constant. The distance from the rotation center to center of mass is defined by $\mathrm{r}_{\mathrm{e}}$, and $\alpha$ defines the angle between the line connecting the center of mass and the platform rotation center, and the line connecting the center of mass and the anchor point. The displacement- and velocity-dependent torque $T(\theta, d \theta / d t)$ results from damping due to a combination of frictional contact between adjacent elements and air damping. For fabricated devices, each beam in the quad-symmetric torsional anchor was $853 \mu \mathrm{m}$ long, $5 \mu \mathrm{m}$ wide, and $20 \mu \mathrm{m}$ deep. Following previous analyses of torsional anchors [16-18], the nonlinear suspension spring constant was calculated through 2-D finite element analysis with ANSYS. The resulting torque-deflection relationship for the spring is accurately described by a third-order polynomial up to a maximum rotation angle of $7^{\circ}$, leading to a nonlinear spring constant described by,

$$
k(\theta)=21820+2307 \theta+1185 \theta^{2}
$$

The damping coefficient $c_{\text {air }}$ of the element was derived according to the shape of the element [19]. For the designs explored in this work, the damping coefficient is given by,

$$
\boldsymbol{c}_{\text {air }}=\frac{3 \pi}{16} \frac{\mu\left(\boldsymbol{R}_{2}^{4}-\boldsymbol{R}_{1}^{4}\right)}{\boldsymbol{h}}
$$

where $\mu$ is the viscosity of air, $h$ is the substrate gap, $R_{2}$ is the outer radius of the leaf element, and $R_{l}$ is an effective inner radius used to account for the notch removed from the leaf to provide connection to the spring. Note that the leading $3 \pi / 16$ factor results from the use of a leaf design which consists of a partial disk covering an angular range of $3 \pi / 4 \mathrm{rad}$. Damping due to the spring beams and connection ring was assumed to be negligible compared to the leaf element. Using this expression, an estimated damping coefficient of $85 \mathrm{pN} \cdot \mathrm{m} \cdot \mathrm{s} / \mathrm{rad}$ was calculated and used for dynamic modeling. To model interfacital friction between adjacent leaf elements, a static friction coefficient of 0.4 and dynamic friction coefficient of 0.3 were found to provide a reasonable match between simulated and experimental results.

Theoretical values of switching times were determined using three methods. First, an analytic approximation was derived by assuming a constant angular velocity during a switching event, so that the direction and magnitude of the frictional contact force remains constant. Under zero initial conditions for leaf displacement and velocity, and assuming a constant angular velocity $\omega_{c}$ during the switching event, Eqn. (1) may be linearized and solved for delay time as,

$$
t_{\text {delay }}=\frac{1}{\omega_{n}} \cos ^{-1}\left[1+\frac{k-\Omega^{2} m r_{c} r_{e} \cos \alpha}{T-\Omega^{2} m r_{c} r_{e} \sin \alpha} \gamma\right]
$$

where $\gamma$ is defined as the critical angle through which the leaf element must rotate before the next element becomes free to move (see Fig. 1), and $\omega_{n}$ is the resonant frequency given by,

$$
\omega_{n}=\sqrt{\frac{k-\Omega^{2} m r_{c} r_{e} \cos \alpha}{m r_{e}^{2}+I}},(-\cos \alpha)>0
$$

and where the linear spring constant $k$ in Eqns. (4) and (5) can be determined by taking the average value of $k(\theta)$ given by Eqn. (2), i.e.

$$
k=\frac{1}{\gamma} \int_{0}^{\gamma} k(\theta) d \theta
$$

While Eqn. (4) provides a simple estimate for the switching time, it ignores significant nonlinearities inherent in the torsional spring and damping parameters. For a more accurate simulation, the full nonlinear equation of motion was solved numerically using Matlab to determine device dynamics.

Finally, while the analytic and numerical solutions provide insight into single-element switching times, the full time delay mechanism involves multiple elements with interfaces which undergo dynamic collisions during a switching event, and thus experience mechanical interactions beyond friction alone. To simulate multi-element dynamic behavior including interfacial impact events, element contact was included in numerical dynamic simulations using ADAMS software. The nonlinear springs were modeled by connecting each element to the chip carrier by a revolute joint, with a non-linear torque applied to each element. Contact constraints were applied between the first and second elements, and between the second and third elements. As discussed previously, friction was included to simulate interfacial damping between mechanism elements.

\section{EXPERIMENTAL DETAILS}

Micro time delay mechanisms were fabricated using a single mask SOI/DRIE process with a $20 \mu \mathrm{m}$ thick device layer and $2 \mu \mathrm{m}$ buried oxide layer. Minimum resolution after DRIE processing was better than $3 \mu \mathrm{m}$ for both lines and spaces, allowing the gap between adjacent mechanism elements to be on the order of 2-3 $\mu \mathrm{m}$. A maximum sidewall roughness of $\mathrm{R}_{\mathrm{a}}=0.01 \mu \mathrm{m}$ was measured parallel to the wafer surface. Following HF release and supercritical drying, devices were stored in ambient laboratory conditions for several weeks prior to testing. Electron micrographs of a single leaf and a closeup of the locking interface between adjacent leafs from a typical fabricated device are shown in Fig. 2.

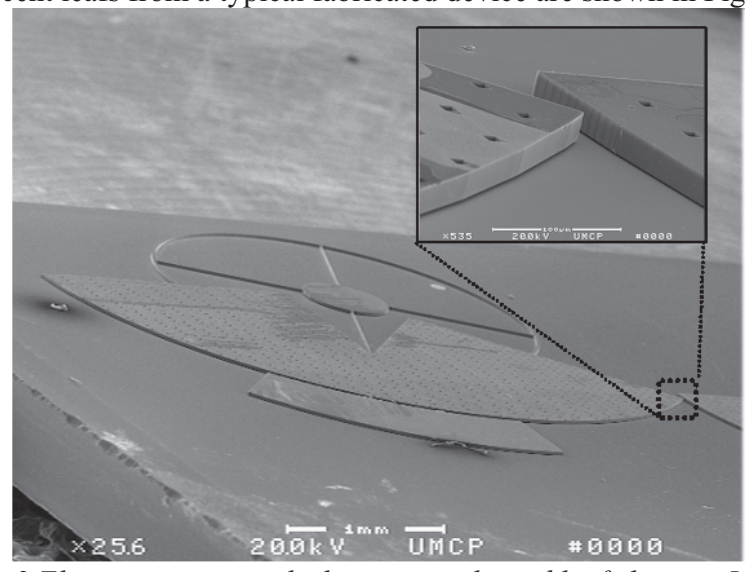

Fig. 2 Electron micrograph showing a released leaf element. Inset details locking interface between adjacent elements.

Devices were designed with $1.1 \mathrm{~mm}$ radius leaf elements positioned with centers of mass located $28 \mathrm{~mm}$ from the center of munition rotation, and to initiate switching at angular speeds ranging from $20 \mathrm{rps}(46 \mathrm{~g})$ to $50 \mathrm{rps}$ ( $285 \mathrm{~g})$. System parameters for each of the four designs are provided in Table 1, including predicted time delays based on multi-body dynamics modeling.

Table 1. Summary of designed critical angles for the first and second elements in fabricated 3-element mechanisms.

\begin{tabular}{r|c|c|c|c} 
Design: & $\# 1$ & $\# 2$ & $\# 3$ & $\# 4$ \\
\hline${\text { critical angle, } 1^{\text {st }} \text { element }(\mathrm{deg})} 1.9$ & 3.1 & 5.1 & 5.6 \\
\hline${\text { critical angle, } 2^{\text {nd }} \text { element }(\mathrm{deg})}$ elem & 1.2 & 1.8 & 3.8 & 4.3 \\
\hline theoretical switching accel. $(\mathrm{g})$ & 34 & 72 & 184 & 223 \\
\hline theoretical delay time $(\mathrm{ms})$ & 1.0 & 0.9 & 0.7 & 0.6
\end{tabular}


Devices were characterized using an experimental consisting of a chip carrier attached to a computer-controlled rotation platform based on a modified photoresist spinner capable of accelerating the chip carrier to speeds of $5000 \mathrm{rpm}$. A rhomboid prism was positioned with one end over the chip, and the other end centered on the rotation axis of the carrier. The rhomboid prism projected the mechanism image to the chip carrier rotation center through two total internal reflections from the end faces of the prism, thereby displacing the chip image by a radial distance defined by the length of the rhomboid prism. Using this approach, a camera positioned over the rotation axis was able to image the chip regardless of the rotation angle of the carrier, enabling long exposure times and eliminating the need for synchronizing image capture from a static camera located at the same radial distance as the chip. A high-speed Phantom v4.0 camera (Vision Research Inc., Wayne, NJ) enabled 3700 frames per second capture with a $0.27 \mathrm{~ms}$ frame interval. Imaging optics were based on a $6.5: 1$ ratio zoom lens (Zoom 6000, Navitar, Rochester, NY) and a 150W fiber optic light source $\left(\mathrm{ACE}^{\circledR} \mathrm{DDL}\right.$, Schott Fostec, Auburn, NY) for coaxial illumination.
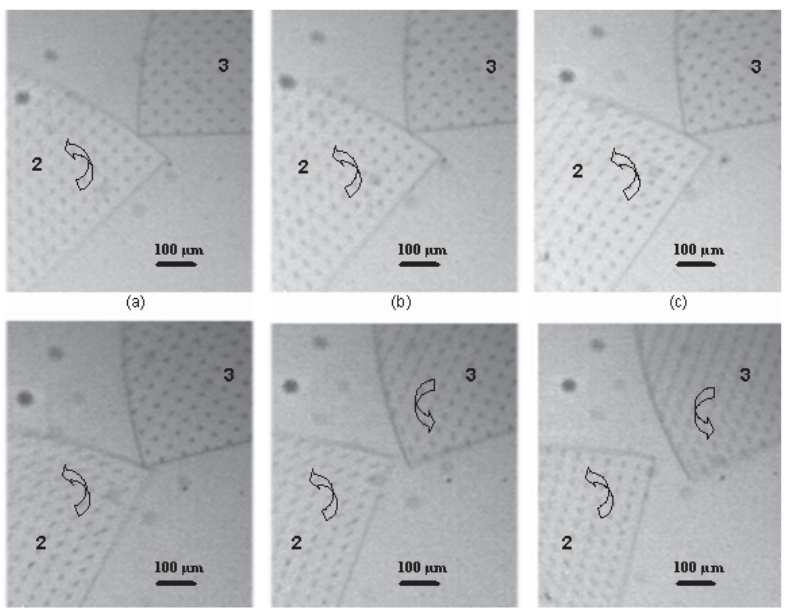

(d)

(e)

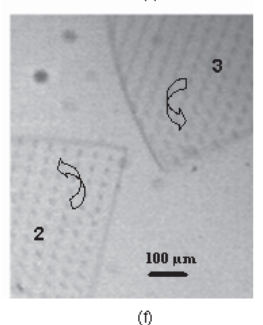

Fig. 3 Switching event between the $2^{\text {nd }}$ and $3^{\text {rd }}$ leaf of a fabricated mechanism. Leaf 3 is constrained from moving in frames (a-d), and released in frames (e-f) after leaf 2 reaches its critical angle.

Fig. 3 shows eight successive images during a typical switching event, revealing the progressive increase in rotation angle for element $\# 2$, and finally the unlocking of element \#3 once the critical angle is reached. Rotation angles were measured using image processing software provided with the camera. Angle measurement tolerance was approximately $0.2^{\circ}$.

Measured centrifugal switching accelerations for each design are shown in Fig. 4, along with the theoretical predictions provided by both analytical modeling and dynamics simulations conducted with ADAMS multi-body dynamics software. Note that while measured variations in switching speed were generally independent of rotation rate, variations in the switching acceleration tended to be proportional to the square of the rotation rate, as shown by the error bars which represent $+/-1 \sigma$ for the experimental data. Although the device designed to switch at the highest rotation speed exhibited slightly lower variability in measured switching acceleration compared to the second-highest speed device, this overall trend remains valid within expected experimental variability. Theoretical predictions are also shown for numerical solutions to the full nonlinear equations of motion, and results of dynamics simulations using ADAMS software. A comparison of experimental and theoretical time delay measurements is shown in Fig. 5. This plot also includes the predicted values from the linearized time delay expression given by Eqn. (4). Error bars reflect the experimental time-measurement resolution for a single camera frame of $+/-0.135 \mathrm{~ms}$. Each experiment was repeated using 3 different devices, with no observed variability in switching time for any of the designs. Overall, the experimental results provide reasonable agreement to the nonlinear analytic model and dynamics simulations for all tested designs. For designs with larger critical angles, the linear analytic model of Eqn. (4) agrees well with experimental results, suggesting that this simple model is suitable for further design optimization without relying on complex numerical solutions or time consuming dynamics simulations.

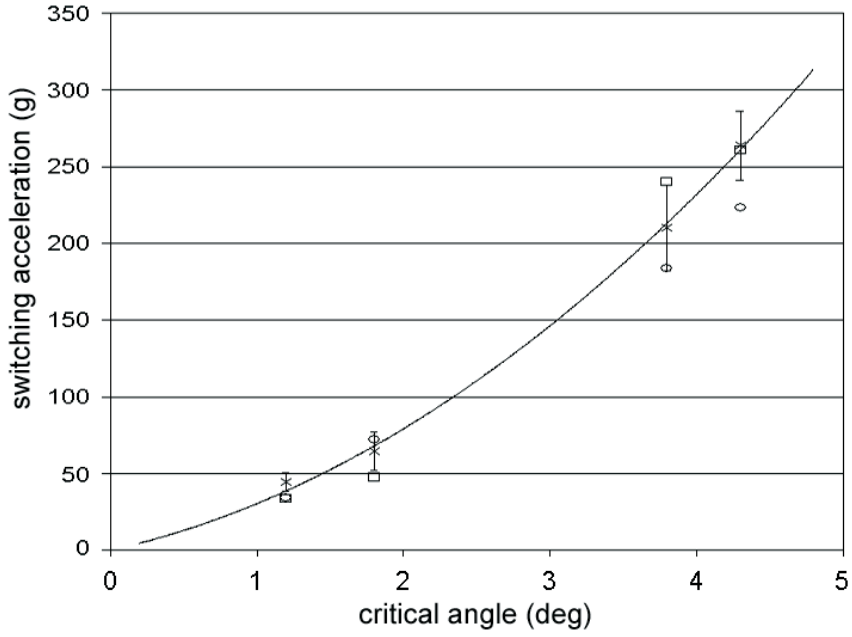

Fig. 4 Comparison of experimental $(\times)$ switching accelerations with nonlinear analytic (o) and dynamics simulation $(\square)$ analyses.

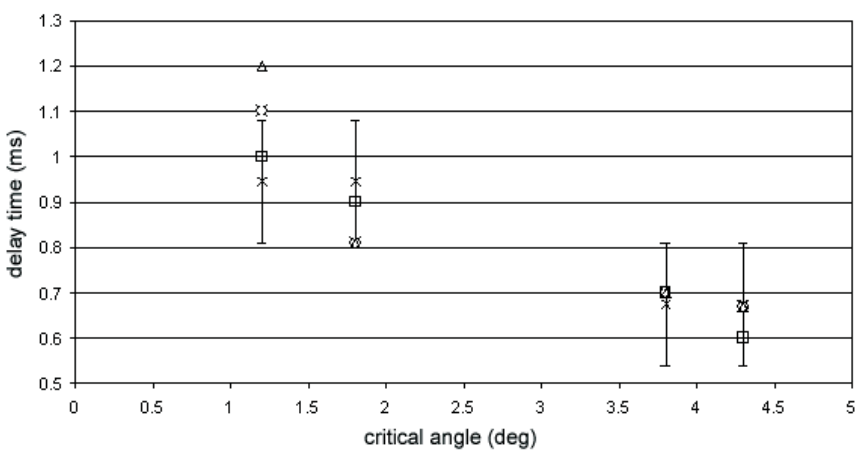

Fig. 5 Comparison of experimental ( $\times$ ), nonlinear numerical (o), linear analytic $(\triangle)$, and dynamics simulation $(\square)$ analyses.

Overall, reasonable agreement was obtained between experimental results and both analytic and dynamics simulation models, within the constraints imposed by the experimental setup. Discrepancies between the different models are reasonable, especially for devices with larger critical angles. Furthermore, such discrepancies are not surprising. The dynamics simulations involved multiple elements with interfaces undergoing dynamic collisions during a switching event. In contrast, interfacial impact was not considered in the analytic models. More importantly, the dynamics simulations used a friction model based on a piecewise continuous relationship between velocity and friction coefficient, while the nonlinear analytic model was simulated using a simpler friction model with discrete static and dynamic friction 
coefficients. Friction is a critical parameter in defining the dynamics of the mechanically-interacting leaf elements, and so the observed discrepancies are not unexpected. Furthermore, despite these discrepancies, agreement between dynamics simulations and nonlinear analytic model results provides confidence that the model captures the key physics of the mechanisms, and is suitable for further use as an analytic tool without relying on the difficult and time consuming dynamics simulations. Additionally, the linearized analytic model converges well with the full nonlinear model for larger critical angles, and provides an accurate prediction of delay time using a simple expression which is suitable as a design tool.

While the sequential-leaf time delay mechanisms fabricated in this work provide up to $0.67 \mathrm{~ms}$ delay per element for a mechanism rotating with a centrifugal acceleration of $263 \mathrm{~g}$, higher switching accelerations are required for many fuzing applications. From the theoretical model, the switching speed can be increased by increasing the stiffness of the suspension spring for the first mechanism element, thereby setting a higher rotation speed threshold required to rotate the first element beyond its critical angle and unlock the remainder of the train. For example, if the spring constant of design \#1 (see Table 1) were increased by a factor of 4, the switching speed would increase from $263 \mathrm{~g}$ to 902 g. It is also desirable to increase the delay time of each element. Delay time is increased by reducing the resonance frequency of the elements, e.g. by reducing the spring constant. Both switching speed and time delay improvements can be realized simultaneously by reducing the spring constants for all mechanism elements, with the exception of the first element, whose suspension spring is stiffened to define a higher initial release acceleration.

\section{CONCLUSIONS}

Leaf-type micro-scale time delay mechanisms have been designed, fabricated, and tested. Delay times as high as $0.95 \mathrm{~ms}$ were realized for single elements within 3-element mechanisms operating at relatively low rotational speeds $(20 \mathrm{rps}$, with a centrifugal acceleration of $44 \mathrm{~g}$ ). At higher speeds (50 rps, $263 \mathrm{~g}$ ) delay time for devices which differed only in their critical rotation angles exhibited delay times of $0.67 \mathrm{~ms}$ per element. This study was conducted to evaluate the basic functionality of microfabricated sequential-leaf time delay mechanisms for application to fuzing systems. Higher rotation speeds of 200-250 rps and associated centrifugal accelerations of several thousand $g$ are required for many munitions applications, with total delay times from tens to hundreds of ms. While the demonstrated time delays and switching accelerations for 3-element mechanisms are not yet sufficient for such applications, both the concept and the analytical model have been validated experimentally in this work, and the models suggests that modified designs are within reach for meeting required application goals.

\section{REFERENCES}

1. MIL-HDBK-757(AR), Fuzes (1994).

2. S. Michaelis, H.-J. Timme, M. Wycisk, and J. Binder, "Additive electroplating technology as a post-CMOS process for the production of MEMS acceleration-critical Switches for transportation applications", J. Micromech. Microeng., 10, 120-123 (2000).

3. T. Tonnesen, O. Ludtke, J. Noetzel, J. Binder, and G. Mader, "Simulation, design and fabrication of electroplated acceleration switches",J. Micromech. Microeng., 7, 237-239 (1997).

4. M. E. Anderson, "An analysis of the runaway escapement as a mechanical acceleration-integrator", M.S. thesis, University of California, Los Angeles, CA (1966).

5. G. Lowen, and F. Tepper, "Dynamics of the pin pallet runaway escapement", Tech. Rep. ADB0289355, Army Armament Research and Development Command, Dover, NJ (1978).

6. A. C. Wang, and T. W. Lee, "On the dynamics of intermittent-motion mechanisms-Part2: geneva mechanisms, ratchets, and escapements", J. Mechanisms, Transmissions, and Automation in Design, 150, 541-551 (1983).

7. L. P. Farace, "A gearless safe and arming device for artillery firing (program summary and mathematical analysis)", Tech. Rep. ADA0412981, Frankford Arsenal, Philadelphia, PA (1975).

8. M. Kenig, "Failure analysis of gearless safety and arming device", Tech. Rep. AD7800147, Frankford Arsenal, Philadelphia, PA (1973).

9. N. Czajkowski, "Theoretical and experimental analysis of the dynamics of Junghan's escapement", M.S. thesis, University of Maryland, College Park (1955).

10. K. Schulgasser, and C. Dock, "Development of the dock escapement", in Proc. of the Timers for Ordnance Symposium, 1, 15-34 (1966).

11. D. Popovitch, "Timing escapement mechanism", U.S. Patent 3168833 (1965).

12. C. H. Robinson, R. H. Wood, and T. Q. Hoang, "Development of inexpensive, ultra-miniature MEMS-based safety and arming (S\&A) device for small-caliber munition fuzes", Proc. 23 "rd Army Science Conference, CO-02 (2002).

13. W. E. Ryan, "Rotary-type setback leaf S\&A mechanisms, analysis and design", Tech. Rep. HDL TR1190 (U-149244), Harry Diamond Laboratory, Adelphi, MD (1964).

14. R. W. Baker, "Analysis of a rotary-type, multiple leaf, setback sensor for a safety and arming mechanism”, M.S. thesis, University of Rhode Island, RI (1977).

15. A. Hausner, "An analysis of friction in a T293 setback leaf system”, Tech. Rep. AD602624, Harry Diamond Labs, Washington D. C. (1960).

16. S. M. Barnes, S. L. Miller, M. S. Rodgers, and F. Bitsie, "Torsional ratcheting actuating system", Proc. MSM, 273-276 (2000).

17. W. Davis, A.P. Pisano, "On the vibrations of a MEMS gyroscope”, Proc. MSM, 557-562 (1998).

18. W. Davis, "Mechanical analysis and design of vibratory micromachined gyroscopes", Ph.D. dissertation, University of California, Berkeley, CA (2001).

19. A. Padmanabhan, "Silicon micromachined sensors and sensor arrays for shear-stress measurements in aerodynamic flows", Ph.D. dissertation, Massachusetts Institute of Technology, Cambridge, MA (1997). 